\title{
WHAT IS THE APPROPRIATE SAMPLE SIZE TO RUN ANALYTIC HIERARCHY PROCESS IN A SURVEY-BASED RESEARCH?
}

\begin{abstract}
Analytic Hierarchy Process (AHP) is often adopted in survey-based research activities and the number of participants involved in AHP studies ranges from few experts to hundreds of interviewed people. A common goal of survey research is to collect data representative of a population and, to this end, determining sample size is essential. The question then is, what is the appropriate sample size to run AHP in a survey-based study? To the best of the authors' knowledge, no previous study addressed the proposed research question in the field of AHP-based survey. The current study aimed to propose a simulation approach for addressing the question of appropriate sample size for AHPbased survey. The proposed approach and the related findings will be presented and discussed.
\end{abstract}

Keywords: Analytic Hierarchy Process, sample size determination, survey study.

\section{Introduction}

Analytic Hierarchy Process (AHP) is often adopted in survey-based research activities and the number of participants involved in AHP studies ranges from few experts to hundreds of interviewed people. A common goal of survey research is to collect data representative of a population and, to this end, determining sample size is essential. The question then is, what is the appropriate sample size to run AHP in a survey-based study?

\section{Literature Review}

To the best of the authors' knowledge, no previous study addressed the proposed research question in the field of AHP-based survey, although there is a literature on sample size determination in survey research (Barlett, 2001). Cochran (1977) proposed an approach based on two key factors: the error the research is willing to accept, referred as margin error, and the level of acceptable risk the researcher is willing to accept that the true margin error exceed the acceptable margin of error, referred as alpha level. In general, a marginal error of 5\% and an alpha level of .05 is acceptable for most research. It is interesting to note that two threads on Researchgate website are dedicated to the specific topic of AHP-based survey

\section{Objectives}

The current study aimed to propose a simulation approach for addressing the question of appropriate sample size for AHP-based survey.

\section{Research Methodology}

We performed simulation experiments, by generating AHP matrix, according to the model adopted by Laininen (2003). Briefly, each value of the matrix is obtained by adding into the expectation a normally distributed random error with the standard 
deviation $\sigma$, and then rounding its exponential to the nearest fraction formed by integers from one to nine. In general, we present simulation results calculated by using the value $\sigma$ $=0.4$ which seems to be typical in experimental AHP-matrices (Leskinen, 2000). For each consistent AHP matrix of size 3 x 3 (i.e., comparing three alternatives A, B and C), we simulated up to 400 experts and we repeated the simulation 1000 times in order to compute the sample size which enable to achieve a margin error of $5 \%$ with an alpha level of 0.05 for each weight estimation.

\section{Data Analysis}

The simulation showed that the sample size needed to achieve a margin error of $5 \%$ with an alpha level of 0.05 varied according to the expected weights from 19 to more than 400 subjects. Smaller sample sizes were required in case of equally important alternative (e.g. with expected weight of 0.333 for all the three alternative the required sample size is 19 ; with expected weight of 0.058 for alternative A, 0.278 for alternative $\mathrm{B}, 0.663$ for alternative $\mathrm{C}$ a sample size larger than 400 is required).

\section{Limitations}

The current study is based on simulated data and on the basic hierarchy adopted in AHP (matrix size $3 \times 3$ ). For that reason, further studies are needed verify the main findings against real data and on more complex hierarchy. Moreover, the absolute value of the margin error could be selected instead of the relative value (e.g., 0.01 instead of $5 \%$ ).

\section{Conclusions}

The current study proposed an approach based on simulated experiment to estimate the sample size needed to achieve a margin error under a defined threshold with an acceptable alpha level.

\section{Key References}

Cochran, W. G. (1977). Sampling techniques. New York: John Wiley \& Sons.

Barlett, J. E., Kotrlik, J. W., \& Higgins, C. C. (2001). Organizational research: Determining appropriate sample size in survey research. Information Technology, Learning, and Performance Journal, 19(1), 43.

Leskinen, P. (2000). Measurement scales and scale independence in the Analytic Hierarchy Process. Journal of Multicriteria Decision Analysis, 9(4), 163.

Laininen, P., \& Hämäläinen, R. P. (2003). Analyzing AHP-matrices by regression. European Journal of Operational Research, 148(3), 514-524. 